\title{
Maximum Likelihood Transmission Image Reconstruction for Overlapping Transmission Beams
}

\author{
Jeffrey A. Fessler, Daniel F. Yu, and Edward P. Ficaro \\ 4240 EECS Bldg., University of Michigan, Ann Arbor, MI 48109-2122
}

\begin{abstract}
In many transmission imaging geometries, the transmitted "beams" of photons overlap on the detector, such that a detector element may record photons that originated in different sources or source locations and thus traversed different paths through the object. Examples include systems based on scanning line sources or on multiple parallel rod sources. The overlap of these beams has been disregarded by both conventional analytical reconstruction methods as well as by previous statistical reconstruction methods. We propose a new algorithm for statistical image reconstruction of attenuation maps that explicitly accounts for overlapping beams in transmission scans. The algorithm is guaranteed to monotonically increase the objective function at each iteration. The availability of this algorithm enables the possibility of deliberately increasing the beam overlap so as to increase count rates. Simulated SPECT transmission scans based on a multiple line source array demonstrate that the proposed method yields improved resolution/noise tradeoffs relative to "conventional" reconstruction algorithms, both statistical and nonstatistical.
\end{abstract}

\section{INTRODUCTION}

To reconstruct quantitatively accurate images of radioisotope emission distributions in SPECT, one must compensate for the effects of photon absorption or attenuation. Accurate attenuation correction requires good attenuation maps, and one can reconstruct such maps from transmission scan measurements obtained either prior to or simultaneously with the SPECT emission scan.

Several source/detector configurations for SPECT transmission scans have been investigated, including a single fixed line source opposite a symmetric fan-beam collimator, used in triple-head SPECT cameras, a scanning line source for orthogonal dual-head cameras, and offset line sources opposite asymmetric fan-beam collimators. Cellar et al [1] describe an alternative geometry based on several fixed-position collimated line sources opposing a parallel-beam collimator. In that system design, the source collimation was selected to minimize overlap on the detector of the transmitted "fanbeams." They then applied the filtered back-projection (FBP) algorithm to reconstruct the attenuation map (an ART algorithm was also mentioned without details). This source collimation has the undesirable consequence of very nonuniform count profiles, as shown in Figure 4 of [1]. It is natural to expect that higher and more uniform count profiles could lead to better reconstructed attenuation maps if the overlap can be properly modeled by the reconstruction method.

In both the scanning line source geometry and the geometry of Celler et al [1], there can be overlap of the beam footprints. Previously published statistical algorithms for transmission tomography, e.g. [2-7], are inapplicable to the multiple source problem when the beams overlap. In this paper we formulate a statistical model for multiple-source transmission measurements with arbitrary overlapping beams, and then derive an iterative algorithm for maximizing the likelihood (or a regularized variant thereof). The log-likelihood is not necessarily globally concave, which usually precludes proofs of convergence to a global maximum. The algorithm that we present is guaranteed to increase the likelihood at every iteration, and the set of fixed points of the algorithm is the same as the set of stationary points of the objective function. The algorithm also satisfies the continuity conditions of Meyer [8]. Therefore, by the convergence results in [8], the proposed algorithm produces a sequence of estimates that converge from any nonnegative initial image to a stationary point of the objective, provided the set of stationary points is not a continuum. This is nearly as strong of a convergence result as one might expect for a possibly nonconcave objective function.

\section{STATistical MODEL}

Let $Y_{i}$ denote the number of photons counted by the $i$ th detector element ${ }^{1}$ during the transmission scan, for

\footnotetext{
${ }^{1}$ Each "detector element" corresponds to a unique radial position and view angle, i.e., for typical 2D reconstruction
} 
$i=1, \ldots, N$, where $N$ is the number of measurement elements. Each detector element conceivably may count photons that originated in any of the $M \geq 1$ sources. We assume that separate blank scans are available for each of the sources (or source positions for a scanning line source). (This information is essential for unscrambling the multiplexing of overlapping beams.) Let $b_{i m}$ denote the mean number of photons that would be observed during a transmission scan by the $i$ th detector originating in the $m$ th source in the absence of any patient in the scanner. Typically the $b_{i m}$ 's would be determined by a periodic calibrating "blank scan", performed separately for each of the $M$ sources, and then scaled by the relative durations of the blank scan and transmission scans. However, we ignore any statistical uncertainty in the $b_{i m}$ 's and treat them as known constants. This assumption is reasonable provided the blank scans are sufficiently lengthy.

Let $\mu^{\text {true }}=\left[\mu_{1}^{\text {true }}, \ldots, \mu_{p}^{\text {true }}\right]^{\prime}$ denote the vector of unknown attenuation coefficients for each of the $p$ pixels or voxels in the attenuation map. The line integral between the $m$ th source and the $i$ th detector location through the attenuating object is approximated by the following sum:

$$
\left[\boldsymbol{A}^{m} \mu\right]_{i}=\sum_{j=1}^{p} a_{i j}^{m} \mu_{j}
$$

where $A^{m}=\left\{a_{i j}^{m}\right\}$ is a $N \times p$ matrix with nonnegative elements and the $a_{i j}^{m}$ 's represent line-lengths or normalized strip-intersection areas ${ }^{2}$. Thus by Beer's law the "survival probability" for a photon transmitted from the $m$ th source in the direction of the $i$ detector is (approximately) $\exp \left(-\left[\boldsymbol{A}^{m} \mu\right]_{i}\right)$.

We assume the $Y_{i}$ 's have independent Poisson distributions:

$$
Y_{i} \sim \text { Poisson }\left\{\bar{y}_{i}\left(\mu^{\text {true }}\right)\right\}
$$

where the means are given by

$$
\bar{y}_{i}(\mu)=\left[\sum_{m=1}^{M} b_{i m} \exp \left(-\left[\boldsymbol{A}^{m} \mu\right]_{i}\right)\right]+r_{i} .
$$

The $r_{i}$ 's are nonnegative constants that one can include to account for the mean contributions of scatter, room background, and emission crosstalk [9]. We treat these $r_{i}$ 's as known constants, though in practice they must be determined experimentally. However, since scatter is a spatially smooth function, one can safely smooth scatter estimates

$N=N_{r} N_{\theta}$ where $N_{r}$ is the number of radial samples along the detector and $N_{\theta}$ is the number of view angles or "steps."

${ }^{2}$ Normalized by strip width fairly heavily, so generally the uncertainty in the $r_{i}$ 's can be made much smaller than that of the $Y_{i}$ 's.

The summation over $m$ in (1) allows for arbitrary overlap of the beams transmitted from each source. Non-overlapping beams would correspond to the assumption that if $b_{i m} \neq 0$, then $b_{i k}=0$ for all $k \neq m$, i.e. $b_{i m} b_{i k}=0$ for all $k \neq m$.

Under the above statistical model, given a particular measurement realization $Y=\left[y_{1}, \ldots, y_{N}\right]^{\prime}$, we can write the log-likelihood for $\mu$ in the following convenient form:

$$
\begin{gathered}
L(\mu, Y)=\sum_{i=1}^{N} h_{i}\left(\sum_{m=1}^{M} u_{i m}(\mu)\right), h_{i}(t)=y_{i} \log t-t, \\
u_{i m}(\mu)=b_{i m} \exp \left(-\left[A^{m} \mu\right]_{i}\right)+r_{i} / M,
\end{gathered}
$$

ignoring constants independent of $\mu$. Since the form of this log-likelihood is sufficiently different from the usual models for emission tomography and transmission tomography [3], previously derived algorithms for maximum likelihood estimation are not directly applicable to this problem.

One could easily derive an expectation-maximization (EM) algorithm [10] that would monotonically increase the likelihood $L(\mu, Y)$ for this problem, generalizing [3]. However, the convergence would be as painfully slow and the M-step as difficult as the usual transmission EM algorithm. Instead, we propose an algorithm based on an extension of our recent work on paraboloidal surrogates methods [2].

Because of the ill-posedness of the reconstruction problem, a penalty term is usually added to the likelihood to encourage piecewise smoothness in the reconstructed image, resulting in the following objective function:

$$
\Phi(\mu, Y)=L(\mu, Y)-\beta R(\mu) .
$$

Our goal is to produce a penalized-likelihood estimate:

$$
\hat{\mu}=\arg \max _{\mu \geq \underline{0}} \Phi(\mu, Y) .
$$

Most roughness penalties $R(\mu)$ can be expressed in the following general form:

$$
R(\mu)=\sum_{k=1}^{K} \psi_{k}\left([\boldsymbol{C} \mu]_{k}\right),
$$

where the $\psi_{k}$ 's are potential functions acting as a norm on the "soft constraints" $C \mu \approx 0$ and $K$ is the number of such constraints. The functions $\psi_{k}$ we consider here are convex, symmetric, nonnegative and differentiable [2]. 


\section{ALGORITHM}

We focus on the unregularized maximum-likelihood problem; the regularized approach easily follows from [2]. Since maximizing the log-likelihood directly is difficult to do, we apply the principle of optimization transfer $[5,11]$ and define a "surrogate function" $Q\left(\mu ; \mu^{n}\right)$ that is easier to maximize. Since this surrogate function depends on the previous estimate $\mu^{n}$ at the $n$th iteration, the algorithm consists of repeatedly maximizing the surrogate function, i.e.

$$
\mu^{n+1}=\arg \max _{\mu \geq \underline{0}} Q\left(\mu ; \mu^{n}\right) .
$$

Note that the maximization is constrained to enforce the nonnegativity constraint. The key algorithm design requirement is to choose $Q$ functions that satisfy the following conditions:

$$
\begin{aligned}
Q\left(\mu^{n} ; \mu^{n}\right) & =L\left(\mu^{n}, Y\right), \forall \mu^{n} \geq \underline{0} \\
\left.\frac{\partial Q}{\partial \mu_{j}}\left(\mu ; \mu^{n}\right)\right|_{\mu=\mu^{n}} & =\left.\frac{\partial L}{\partial \mu_{j}}(\mu)\right|_{\mu=\mu^{n}}, \forall j=1, \ldots, p \\
Q\left(\mu ; \mu^{n}\right) & \leq L(\mu, Y), \forall \mu \geq \underline{0} .
\end{aligned}
$$

These conditions ensure that the proposed iteration monotonically increases the likelihood.

A difficulty in maximizing $L$ is the sum over $m$ within the logarithm in (2). To move the summation outside of the logarithm, we first adapt De Pierro's multiplicative convexity trick [12]. Because $h_{i}$ is concave:

$$
\begin{aligned}
h_{i}\left(\sum_{m=1}^{M} u_{i m}(\mu)\right) & =h_{i}\left(\sum_{m=1}^{M} \frac{u_{i m}^{n}}{\bar{y}_{i}^{n}} \frac{u_{i m}(\mu)}{u_{i m}^{n}} \bar{y}_{i}^{n}\right) \\
& \geq \sum_{m=1}^{M} \frac{u_{i m}^{n}}{\bar{y}_{i}^{n}} h_{i}\left(\frac{u_{i m}(\mu)}{u_{i m}^{n}} \bar{y}_{i}^{n}\right)
\end{aligned}
$$

where $u_{i m}^{n} \triangleq u_{i m}\left(\mu^{n}\right)$, and $\bar{y}_{i}^{n} \triangleq \bar{y}_{i}\left(\mu^{n}\right)$. This inequality leads to our first surrogate function:

$$
\begin{aligned}
Q_{1}\left(\mu ; \mu^{n}\right) & \triangleq \sum_{i=1}^{N} \sum_{m=1}^{M} \frac{u_{i m}^{n}}{\bar{y}_{i}^{n}} h_{i}\left(\frac{u_{i m}(\mu)}{u_{i m}^{n}} \bar{y}_{i}^{n}\right) \\
& =\sum_{i=1}^{N} \sum_{m=1}^{M} \frac{u_{i m}^{n}}{\bar{y}_{i}^{n}} g_{i m}^{n}\left(\left[A^{m} \mu\right]_{i}\right),
\end{aligned}
$$

where

$$
\begin{aligned}
b_{i m}^{n} & \triangleq \frac{\bar{y}_{i}^{n}}{u_{i m}^{n}} b_{i m}, \\
r_{i m}^{n} & \triangleq \frac{\bar{y}_{i}^{n}}{u_{i m}^{n}} \frac{r_{i}}{M} \\
g_{i m}^{n}(l) & \triangleq y_{i} \log \left(b_{i m}^{n} e^{-l}+r_{i m}^{n}\right)-\left(b_{i m}^{n} e^{-l}+r_{i m}^{n}\right) .
\end{aligned}
$$

The surrogate function $Q_{1}$ remains too difficult to maximize directly because the argument of each $h_{i}$ still depends on $u_{i m}$, which has a complicated exponential form. However, it follows easily from the results in $[2,13]$ that the following paraboloidal function is a valid surrogate for $Q_{1}$ :

$Q_{2}\left(\mu ; \mu^{n}\right) \triangleq \sum_{i=1}^{N} \sum_{m=1}^{M} \frac{u_{i m}^{n}}{\bar{y}_{i}^{n}} q_{i m}^{n}\left(\left[A^{m} \mu\right]_{i}\right) \triangleq Q\left(\mu ; \mu^{n}\right)$

where

$q_{i m}^{n}(l) \triangleq g_{i m}^{n}\left(l_{i m}^{n}\right)+\dot{g}_{i m}^{n}\left(l_{i m}^{n}\right)\left(l-l_{i m}^{n}\right)-\frac{1}{2} c_{i m}^{n}\left(l-l_{i m}^{n}\right)^{2}$

and

$$
\begin{aligned}
\dot{g}_{i m}^{n}(l) & \triangleq \frac{d}{d l} g_{i m}^{n}(l) \\
\ddot{g}_{i m}^{n}(l) & \triangleq \frac{d^{2}}{d l^{2}} g_{i m}^{n}(l) \\
l_{i m}^{n}=\left[A^{m} \mu^{n}\right]_{i} & =\sum_{j=1}^{p} A_{i j}^{m} \mu_{j}^{n} .
\end{aligned}
$$

To ensure (7), we must choose the curvatures $\left\{c_{i m}^{n}\right\}$ appropriately $[2,13]$. As discussed in [2], for the fastest convergence rate, we would like to choose the curvatures as small as possible, subject to the constraint that the surrogate function $q_{i m}^{n}$ lies below the functions $g_{i m}^{n}$ (see [2, Eqn. 16] for the formula for the optimum curvature).

Since our second surrogate $Q_{2}$ is a quadratic functional, it is easily maximized by a variety of algorithms, including the coordinate ascent algorithm $[4,14]$. Adding a penalty function is straightforward.

To obtain a monotonic algorithm that converges relatively quickly, we can apply coordinate ascent to the surrogate $Q$ defined in (12), i.e., sequentially update one pixel at a time while holding all other pixels fixed. The maximization step of the coordinate ascent for pixel $j$ is ${ }^{3}$ :

$$
\begin{aligned}
\hat{\mu}_{j}^{\text {new }} & =\arg \max _{\mu_{j} \geq 0} \hat{Q}_{j}^{n}\left(\mu_{j}\right)-\beta \hat{R}_{j}\left(\mu_{j}\right) \\
& =\left[\hat{\mu}_{j}+\frac{\dot{Q}_{j}^{n}(\hat{\mu})-\beta \dot{R}_{j}(\hat{\mu})}{d_{j}^{n}+\beta \hat{p}_{j}}\right]_{+},
\end{aligned}
$$

where

$$
\begin{aligned}
\dot{Q}_{j}^{n}(\hat{\mu}) & \triangleq \sum_{i=1}^{N} \sum_{m=1}^{M} \frac{u_{i m}^{n}}{\bar{y}_{i}^{n}} a_{i j}^{m}\left[\dot{g}_{i m}^{n}\left(l_{i m}^{n}\right)-c_{i m}^{n}\left(\hat{l}_{i m}-l_{i m}^{n}\right)\right] \\
\hat{l}_{i m} & \triangleq\left[\boldsymbol{A}^{m} \hat{\mu}\right]_{i} \sum_{j=1}^{p} \boldsymbol{A}_{i j}^{m} \hat{\mu}_{j}
\end{aligned}
$$

\footnotetext{
${ }^{3}$ For a detailed derivation, refer to [2].
} 


$$
\begin{aligned}
\dot{g}_{i m}^{n}(l) & =\left(\frac{y_{i}}{b_{i m}^{n} e^{-l}+r_{i m}^{n}}-1\right) b_{i m}^{n} e^{-l} \\
d_{j}^{n} & \triangleq \sum_{i=1}^{N} \sum_{m=1}^{M} \frac{u_{i m}^{n}}{\bar{y}_{i}^{n}}\left(a_{i j}^{m}\right)^{2} c_{i m}^{n},
\end{aligned}
$$

and $\hat{R}_{j}\left(\mu_{j}\right)$ denotes the penalty surrogate parabola for $\mu_{j}$, and $\hat{p}_{j}$ denotes its curvature. Because of our construction based on surrogate functions that satisfy (7), this update is guaranteed to monotonically increase the value of $\Phi$. One iteration is finished when all pixels are updated via (14) in a sequential order. We update the paraboloidal surrogate function after one iteration of coordinate ascent (CA), although one could also perform more than one $\mathrm{CA}$ iteration per surrogate.

\section{Simulation RESULTS}

We compared the proposed reconstruction algorithm to the "conventional" reconstruction algorithms (statistical and FBP) that treat the transmission measurements simply as ideal normalized parallel "strip-integrals". The system geometry corresponded fairly closely to the Siemens Profile ${ }^{\text {TM }}$ system (Hoffman Estates, IL) [15]. The sources for the simulated system consisted of a multiple line source array with 14 sources, unequally spaced, located on a line parallel to the detector and 110 $\mathrm{cm}$ away from the detector plane. The detector plane was located $22 \mathrm{~cm}$ away from the center of rotation. The source collimation in our simulations is $2.6^{\circ}$. The image consisted $128 \times 128$ pixels of size $3.56 \times 3.56 \mathrm{~mm}^{2}$. The sinogram size was $128 \times 60$ with detector bins of width $4.8 \mathrm{~mm}$ (i.e., the simulated detector response was rectangular with width $4.8 \mathrm{~mm}$ ). We performed the simulation with 523,000 transmitted counts, and 263,000 background counts. For simplicity, we used a space-invariant quadratic penalty over first-order neighbors throughout our simulations. Figure 1 shows the phantom used in our simulations.

Figures 2 shows reconstructions of noisy data using FBP, the parallel algorithm ${ }^{4}$, and the proposed algorithm. The spatial resolution of the images in these figures is 4.7 pixels on the left column and 6.8 pixels on the right column.

We observe from Figure 2 that the proposed algorithm produces less noisy reconstructions than both the parallel algorithm and FBP. Since the parallel algorithm is based on an incorrect system and statistical model, one expects

\footnotetext{
${ }^{4}$ We refer to the penalized-likelihood reconstruction assuming ideal normalized parallel "strip-integrals" (and ignoring beam overlap) as the parallel algorithm.
}

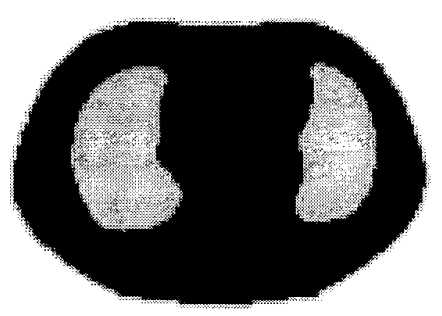

Fig. 1 Digital Phantom used in our simulations

artifacts due to model mismatch. The absence of apparent artifacts in Figure 2 is due to regularization and noise. Figure 3 shows the reconstructed images from noiseless data ( $4.6^{\circ}$ collimation angle) using the parallel and proposed algorithms with almost no regularization $\left(\beta=2^{-10}\right)$. The reconstructed image from noiseless data using the parallel algorithm shows severe artifacts resulting from model mismatch, which are absent in the reconstructed image from noiseless data using the proposed algorithm (Figure 3b).

We also performed studies of optimal collimation angles for a given system. (Due to space constraints, we only outline our conclusions.) We found that the proposed algorithm outperforms the parallel algorithm at all collimation angles, but the optimal collimation angle for the proposed algorithm is larger than the parallel algorithm. At large collimation angles, the proposed algorithm produced reconstructions with significantly smaller variances than the parallel algorithm, given a fixed spatial resolution. Thus it is desirable to open up the source collimators and allow beam overlap - provided the overlap is modeled appropriately in the reconstruction algorithm.

\section{CONCLUSION}

We have presented a new algorithm for statistical image reconstruction of attenuation maps that explicitly accounts for overlapping beams in transmission scans. The algorithm is guaranteed to monotonically increase the objective function at each iteration, and achieves better variance/resolution tradeoffs than "conventional" image reconstruction algorithms, both statistical (the parallel algorithm) and non-statistical (FBP). However, the proposed algorithm is more time-consuming and uses more memory than conventional statistical algorithms. For simulations we performed, the time that the proposed algorithm took was about 3.3 times that of the parallel algorithm. 

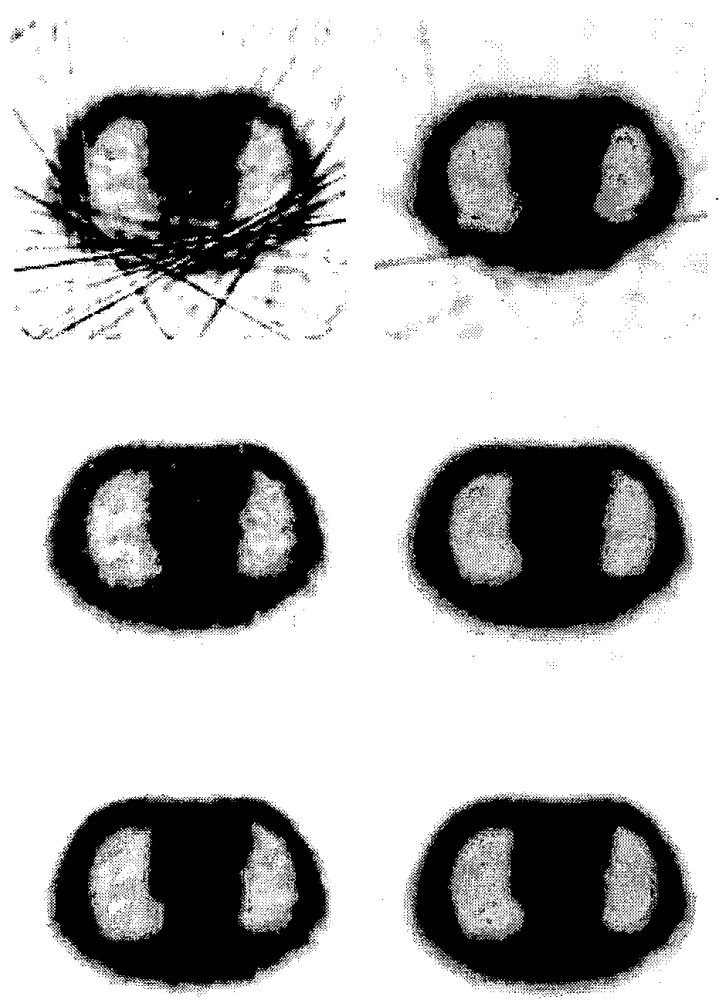

Fig. 2 New sources; collimation angle: $2.6^{\circ} ; 785,000$ counts; left column: resolution 4.7 pixels; right column: resolution 6.8 pixels; top row: FBP; middle row: parallel algorithm; bottom row: proposed algorithm.

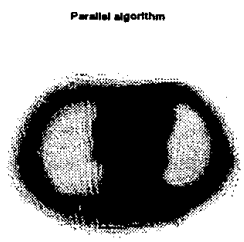

(a)

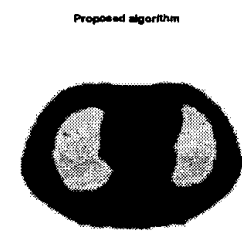

(b)
Fig. 3 Reconstruction using the parallel and proposed algorithms with almost no regularization; collimation angle $4.6^{\circ}$ (a) Parallel algorithm (b) Proposed algorithm.

\section{ACKNOWLEDGMENT}

The authors gratefully acknowledge Peggy Hawman for assistance with the Siemens Profile geometrical specifications.

\section{REFERENCES}

[1] A. Celler, A. Sitek, and R. Harrop, "Reconstruction of multiple line source attenuation maps," IEEE Tr. Nuc. Sci., vol. 44, no. 4, pp. 1503-8, August 1997.

[2] H. Erdoğan and J. A. Fessler, "Fast monotonic algorithms for transmission tomography," IEEE Tr. Med. Im., 1998. To appear.

[3] K. Lange and R. Carson, "EM reconstruction algorithms for emission and transmission tomography," J. Comp. Assisted Tomo., vol. 8, no. 2, pp. 306-316, April 1984.

[4] K. Sauer and C. Bouman, "A local update strategy for iterative reconstruction from projections," IEEE Tr. Sig. Proc., vol. 41, no. 2, pp. 534-548, February 1993.

[5] K. Lange and J. A. Fessler, "Globally convergent algorithms for maximum a posteriori transmission tomography," IEEE Tr. Im. Proc., vol. 4, no. 10, pp. 1430-8, October 1995 .

[6] E. U. Mumcuoglu, R. Leahy, S. R. Cherry, and Z. Zhou, "Fast gradient-based methods for Bayesian reconstruction of transmission and emission PET images," IEEE Tr. Med. Im., vol. 13, no. 3, pp. 687-701, December 1994.

[7] J. A. Fessler, "Grouped coordinate descent algorithms for robust edge-preserving image restoration," in Proc. SPIE 307I, Im. Recon. and Restor. II, pp. 184-94, 1997.

[8] R. R. Meyer, "Sufficient conditions for the convergence of monotonic mathematical programming algorithms," $J$. Comput. System. Sci., vol. 12, pp. 108-21, 1976.

[9] E. P. Ficaro, J. A. Fessler, P. D. Shreve, J. N. Kritzman, P. A. Rose, and J. R. Corbett, "Simultaneous transmission/emission myocardial perfusion tomography: Diagnostic accuracy of attenuation-corrected $99 \mathrm{~m}$-TcSestamibi SPECT," Circulation, vol. 93, no. 3, pp. 46373, February 1996.

[10] A. P. Dempster, N. M. Laird, and D. B. Rubin, "Maximum likelihood from incomplete data via the EM algorithm," $J$. Royal Stat. Soc. Ser. B, vol. 39, no. 1, pp. 1-38, 1977.

[11] M. P. Becker, I. Yang, and K. Lange, "EM algorithms without missing data," Stat. Meth. Med. Res., vol. 6, no. 1, pp. 38-54, 1997.

[12] A. R. De Pierro, "A modified expectation maximization algorithm for penalized likelihood estimation in emission tomography," IEEE Tr. Med. Im., vol. 14, no. 1, pp. 132137, March 1995.

[13] H. Erdoğan and J. A. Fessler, "Accelerated monotonic algorithms for transmission tomography," in Proc. IEEE Intl. Conf. on Image Processing, volume 2, pp. 680-4, 1998.

[14] J. A. Fessler, "Penalized weighted least-squares image reconstruction for positron emission tomography," IEEE Tr. Med. Im., vol. 13, no. 2, pp. 290-300, June 1994.

[15] E. P. Ficaro, J. A. Fessler, J. N. Kritzman, P. C. Hawman, S. W. DeBruin, and J. R. Corbett, "Multiple line source array TCT/ECT system: A technical phantom evaluation," J. Nuc. Med. (Abs. Book), vol. 40, no. 5, pp. 4, May 1999.

This work was supported in part by NIH grants CA60711 and CA-54362. 